Review

\title{
Novel Approaches to Vaccine Delivery
}

\author{
Derek T. O'Hagan ${ }^{1,3}$ and Rino Rappuoli²
}

\begin{abstract}
Received February 12, 2004; accepted May 11, 2004
Although the currently available vaccines represent an outstanding success story in modern medicine and have had a dramatic effect on morbidity and mortality worldwide, it is clear that improvements are required in the current vaccine delivery technologies. Improvements are required to enable the successful development of vaccines against infectious diseases that have so far proven difficult to control with conventional approaches. Improvements may include the addition of novel injectable adjuvants or the use of novel routes of delivery, including mucosal immunization. Mucosal delivery may be required to provide protection against pathogens that infect at mucosal sites, including sexually transmitted diseases. Alternatively, novel approaches to delivery, including mucosal administration, may be used to improve compliance for existing vaccines. Of particular interest for safer mass immunization campaigns are needle-free delivery devices, which would avoid problems due to needle re-use in many parts of the world and would avoid needle-stick injuries.
\end{abstract}

KEY WORDS: adjuvants; emulsions; mucosal delivery; microparticles; needle-free immunization;vaccine delivery.

"An ounce of prevention is worth a pound of cure."

-Benjamin Franklin

\section{INTRODUCTION: VACCINES REPRESENT AN OUTSTANDING SUCCESS STORY IN MODERN MEDICINE}

The introduction of vaccines into medical practice at the beginning of the past century has had an extraordinary impact on human health and welfare and represents an unparalleled success story in modern medicine. Vaccines are considered to be the safest and most effective medical intervention currently available. In conjunction with the introduction of antibiotics and modern hygiene practices, vaccines have contributed enormously to a steady decline in the mortality and morbidity caused by infectious diseases (Table I). Each year, the currently available vaccines prevent up to 3 million deaths and $>750,000$ children are protected from serious disability. Nevertheless, the issue of vaccine safety has been with us since vaccines were first introduced and is an issue that is here to stay (1). Although vaccines have been used safely for many decades, there has been a relatively recent shift in the public perception of their safety. Paradoxically, the heightened concerns about vaccine safety are largely a consequence of their success. Due to the success of vaccination policy, the level of vaccine-preventable disease in many Western societies is now very low. As a consequence, some parents now claim that their children no longer need to be vaccinated. However, this approach ignores societal responsibilities and fails to appreciate the crucial role of "herd immunity," which prevents the

\footnotetext{
${ }^{1}$ Chiron Vaccines, Emeryville, California 94608, USA.

${ }^{2}$ Chiron Vaccines, 53100 Siena, Italy.

${ }^{3}$ To whom correspondence should be addressed. (e-mail: Derek O’Hagan@chiron.com)
}

free circulation of pathogens and protects those most susceptible to serious disease following infection. In addition, a decision not to vaccinate exposes the individual child to a much higher risk for infection if organisms begin to recirculate in the community.

While a claim of complete safety is impossible for any medical intervention, with almost 100 years of data and billions of doses, vaccines have an exemplary safety profile. However, since vaccines are administered to millions of infants annually, it is clear that the level of scrutiny of vaccines will continue to be intense. Therefore, the safety hurdles applied to new vaccines and delivery approaches will be high, with rigorous evaluations the correct and established practice. Any new delivery technology will need to prove beyond reasonable doubt that vaccine potency is not impaired in comparison to the established vaccine. While this might be achievable when the correlates of protection for the vaccine are well established, this is not always the case. In addition, the size of the patient population in which non-inferiority needs to be proven may be large and may require a range of individuals from diverse ethnic backgrounds.

\section{THE NEED FOR NEW AND IMPROVED VACCINES}

Despite the success of vaccines, there is a clear need for the development of new vaccines against infectious diseases for which none are yet available, or are inadequate, including HIV, HCV, Neisseria meningitides type B, tuberculosis (TB), and malaria. Unfortunately, these pathogens have proven exceptionally difficult to control using traditional approaches to vaccine development and novel approaches will be needed. Vaccines are also needed to protect against emerging or re- 
Table I. The Impact of Vaccine on Disease Burden in the United States

\begin{tabular}{lrrc}
\hline \multicolumn{1}{c}{ Disease } & $\begin{array}{c}\text { Max. no. cases } \\
\text { (year) }\end{array}$ & $\begin{array}{c}\text { Cases } \\
\text { in } 2001\end{array}$ & $\begin{array}{c}\text { Reduction } \\
\text { in disease }\end{array}$ \\
\hline Smallpox & $48,164(1901)$ & 0 & $100 \%$ \\
Diphtheria & $206,939(1921)$ & 2 & $99.99 \%$ \\
Pertussis & $265,269(1934)$ & 4788 & $98.20 \%$ \\
Tetanus & $1560(1923)$ & 26 & $98.34 \%$ \\
Polio & $21,269(1952)$ & 0 & $100 \%$ \\
Measles & $894,134(1941)$ & 96 & $99.99 \%$ \\
Rubella & $57,686(1969)$ & 19 & $99.97 \%$ \\
Mumps & $152,209(1968)$ & 216 & $99.86 \%$ \\
Hemophilus influenzae & $20,000(1992)$ & 51 & $99.75 \%$ \\
\hline
\end{tabular}

emerging infectious diseases, including West Nile, SARS, Ebola, Hanta and Dengue viruses. In addition, improved vaccines are needed to protect against the threat of pandemic strains of influenza virus and the continued growth and spread of antimicrobial resistant organisms. In addition, vaccines may be required to protect against the threat of bioterrorism (2). Moreover, there is an increasing awareness that infectious agents can cause chronic diseases, which might be prevented or treated with novel vaccines (Table II). Hence, vaccines may also be considered as potential therapeutic agents to treat established infections. It is clear that novel vaccine delivery technologies will be required to enable the development of these new vaccines, particularly those designed as therapies against chronic infections or cancers. The safety profiles of therapeutic vaccines may look very different from traditional vaccines, which are used to protect against infection. Therapeutic vaccines, particularly if used in an oncology setting, or to treat an established life-threatening infectious disease, would most likely be able to sustain a higher level of adverse events without damaging the marketability of the product.

\section{NOVEL APPROACHES TO VACCINE DELIVERY}

Vaccine delivery is represented by a diverse range of technologies and approaches, which are linked by the objective of improving vaccine performance or potency (Table III). Improvements in vaccine delivery may be achieved by the inclusion of components designed to make vaccines more immunogenic (adjuvants), by technologies that allow them to be administered by mucosal routes, or by devices that allow them to be delivered through the skin without the use of needles. Vaccine delivery may be used to improve patient compliance by using a non-invasive route of delivery. This could result in

Table II. Selected Infectious Agents That Are Known to Cause Chronic Diseases

\begin{tabular}{ll}
\hline \multicolumn{1}{c}{ Microorganism } & \multicolumn{1}{c}{ Disease } \\
\hline Hepatitis B and C viruses & Hepatocellular carcinoma \\
Human papilloma virus & Cervical, anal, and vulvar cancer \\
Epstein-Barr virus & Burkitt lymphoma \\
Borrelia burgdorferi & Lyme disease \\
Helicobacter pylori & Peptic ulcer, gastric cancer \\
Human herpes virus B & Kaposi sarcoma \\
HTLV-1 & Adult T-cell lymphoma \\
\hline
\end{tabular}

Table III. Examples of Alternative Approaches to Vaccine Delivery

- Antigen delivery system/adjuvants: alum, MF59, PLG, liposomes, virosomes, etc.

- Adjuvant delivery systems: PLG, MF59, Iscoms, etc.

- DNA vaccine delivery systems: PLG, gene gun, alphaviruses, etc.

- Nonreplicating viral vectors: alphaviruses, etc.

- Live bacterial and viral vectors: Salmonella, adenovirus, etc.

- Intranasal vaccines: LT mutants and/or bioadhesive polymers, etc.

- Oral vaccines: LT mutants, enteric-coated formulations, etc.

- Topical vaccines: vaccine patches (lomai).

- Microneedles: OnVax (Becton Dickinson), Macroflux (Alza), etc.

- Needle-free devices: Epidermal Powder Immunization (Powderject), Biojector, etc.

a significant improvement in an existing vaccine by ensuring that the vaccines are used more broadly. It is likely that vaccine compliance could be significantly improved if vaccines could be administered without the use of needles, since needle-phobia remains a common problem.

Mucosal administration may be required to enable the development of vaccines that may not be possible using conventional routes (e.g., vaccines against sexually transmitted diseases including HIV). In addition, vaccine delivery systems and adjuvants may also be required to improve the immunogenicity of vaccines containing non-traditional antigens, including recombinant proteins, DNA or peptides. This review will summarize recent developments in non-living vaccine delivery systems and devices, and will consider their suitability for use in a wide range of individuals, including pediatric populations. Live replicating approaches to vaccine delivery and nonreplicating viral vectors such as alphaviruses are beyond the scope of this review, but have been discussed in detail elsewhere (3).

\section{Adjuvants and Delivery Systems for Administration by Injection}

Vaccine adjuvants were first described by Ramon about 80 years ago (4) and have been used to improve the immunogenicity of non-living vaccines ever since. Although the principal role of an adjuvant is to improve the immunogenicity of antigens, they can also be used for a variety of more specific purposes (Table IV). Adjuvants have been crucial to the development of vaccines and are likely to prove even more important in the future. The original approaches to the development of vaccines often involved live attenuated or

Table IV. The Role of Adjuvants in Vaccine Development

1. Increase the total titer or functional titers (e.g. bactericidal or neutralizing antibodies, etc.).

2. Decrease the dose of antigen.

3. Decrease the total number of doses.

4. Overcome competition in combination vaccines.

5. Enhance responses in the young or old.

6. Increase the speed and duration of the response.

7. Induce potent cell-mediated immunity.

8. Induce mucosal immunity.

9. Enable successful vaccine development. 
whole inactivated organisms. Live attenuated vaccines did not need adjuvants, since they were able to replicate and produced high levels of antigens and immunostimulatory components. However, most of the inactivated whole cell or purified subunit vaccines did require adjuvants. Although these vaccines were potently immunogenic, since they contained many or all of the components of infectious organisms, they typically induced a significant level of reactogenicity. Consequently, in recent years, there has been a strong tendency to move away from the development of whole organism based vaccines toward a more "minimalist" approach. The majority of vaccines currently under investigation represent highly purified subunit components of pathogens. Unfortunately, these highly purified vaccines lack most of the features of the original pathogen and are usually poorly immunogenic. Hence, the need for vaccine adjuvants to improve immunogenicity is clear. Furthermore, in a mirror image of the shift from impure to more highly purified antigens, the ideal adjuvant strategy is to add back only highly purified or synthetically produced components which will specifically activate the elements of the immune response required for protection, and will not trigger more generalized nonspecific responses.

Although the terms vaccine "adjuvants" and "delivery systems" have often been used interchangeably, a clear distinction can often be made and the respective roles of each can be more clearly defined (Table V). Classically, adjuvants have been defined by what they do, which is to enhance the immune responses to antigens, rather than by how they achieve this. This has resulted in many diverse agents being described as adjuvants, without much appreciation of the mechanisms of how they do this. However, there is a growing appreciation of the important role of the innate immune response in controlling and guiding the antigen specific adaptive response. Recognition of the importance of innate immunity has coincided with an increasing awareness that many classic adjuvants are components of pathogens which are known to activate cells of the innate immune system, including antigen presenting cells (APC), such as dendritic cells (DC). Hence, It is becoming clear that many well known adjuvants, including monophosphoryl lipid A (MPL), double-stranded RNA (dsRNA), lipopolysaccharide (LPS), lipoproteins (LPP), and bacterial DNA (which is rich in $\mathrm{CpG}$ oligo sequences) are effective due to their ability to be recognized by and activate the pathogen recognition receptors (PRR), which are present on innate immune cells, including the Toll-like receptor family (5-7). Adjuvants activate immune responses because they represent pathogen associated molecular patterns (PAMPs),

Table V. Alternative Adjuvant Approaches

\begin{tabular}{ll}
\hline \multicolumn{1}{c}{ Antigen delivery systems } & \multicolumn{1}{c}{ Immunopotentiators } \\
\hline - Insoluble aluminium salts & - MPL and synthetic derivatives \\
- Calcium phosphate & - MDP and derivatives \\
- Liposomes & - Oligonucleotides (CpG, etc.) \\
- Virosomes & - Double-stranded RNA (dsRNA) \\
- Iscoms & - Alternative pathogen-associated \\
- Microparticles (e.g., PLG) & molecular patterns (PAMPs) \\
- Emulsions (e.g., MF59) & - Quils \\
- Virus-like particles & - Small-molecule immune \\
& potentiators (SMIPs) (e.g., \\
& resiquimod [R848] \\
\hline
\end{tabular}

which are highly conserved in a broad range of pathogens, but are absent from multicellular organisms. The growing appreciation that adjuvants represent structural features of pathogens (PAMPs), which are recognized by PRR has resulted in significant efforts to develop optimal synthetic adjuvants from diverse sources, including small molecules (8).

The most commonly used adjuvant, alum, induces a Th2 immune response, which is particularly associated with the production of the cytokine IL-4, and IgG1 and IgE antibodies in mice. Alum is a successful adjuvant for vaccines that are effective due to the induction of antibody responses. For example, alum is a potent adjuvant for the induction of antibodies that neutralize the effects of bacterial toxins (e.g., tetanus and diphtheria). Nevertheless, a Th1 response, which is associated with the induction of gamma interferon and cytotoxic T lymphocytes (CTL), will be required to clear the body of intracellular viral infections. Encouragingly, bacterial DNA, a PAMP, which can be replaced with synthetic oligonucleotides with specific sequences surrounding $\mathrm{CpG}$, appears to be very effective for the induction of potent Th1 responses. Consequently, this adjuvant may have an important role to play in the development of vaccines against intracellular viral pathogens, including HIV and HCV. It is possible that some adjuvants may prove sufficiently potent to allow the eradication of an established viral infection and may enable the successful development of therapeutic vaccines against chronic infections. However, no such vaccines have yet been developed, although many are undergoing extensive evaluations.

To better understand the mechanism of adjuvants and to clarify their roles in vaccine development, PAMPs and related compounds can be called immunopotentiators (Table $\mathrm{V})$. This is helpful, since it allows a clear distinction to be drawn between immunopotentiators and an alternative group of adjuvants that are particulate (e.g., emulsions, liposomes, virosomes, iscoms, virus-like particles and microparticles), and function mainly to promote the uptake of associated antigens into the APC (9). If this classification is linked to the geographical concept of immune reactivity, in which antigens that do not reach local lymph nodes do not induce responses (10), it allows a clearer definition of the role of different adjuvants. The role of a "delivery" based adjuvant is to enhance the amount of antigen reaching the cells responsible for immune response induction, while immunopotentiators activate the cells through interaction with their receptors, including the PRR. Nevertheless, these simple definitions become insufficient when immunopotentiators are included into delivery systems. Immunopotentiators are formulated into delivery systems to focus their effects onto the APC, to maximize their potency, and to minimize their effects on nonimmune cells. Hence, delivery systems can improve the therapeutic ratio of adjuvants, reduce the dose needed, and improve their specificity and safety. Optimal new generation vaccines are likely to comprise recombinant antigens used in conjunction with immunopotentiators and delivery systems for both antigens and adjuvant (Fig. 1).

\section{Alternative Particulate Delivery Systems}

The main hurdle to the development of new and improved vaccine adjuvants has always been safety. Since vaccines are used in healthy individuals, including pediatric 


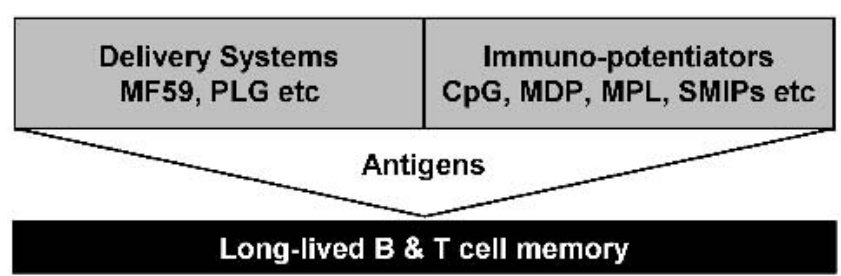

Fig. 1. The optimal composition of new-generation vaccines.

populations, they will need to induce minimal adverse effects to prove acceptable. Hence, although many adjuvants have been extensively evaluated, only insoluble aluminum salts (generically called "alum") have been successfully licensed in North America $(11,12)$. However, an alternative adjuvant, a microemulsion called MF59 (13), was introduced onto the market in Italy in 1997 to be used with an influenza vaccine (Fluad). Fluad subsequently achieved approval throughout Europe in 2000 through the mutual recognition procedure.

\section{The Microemulsion MF59}

Although the exact mechanism of action of MF59 adjuvant remains to be defined, it appears to function as a delivery system and promotes the uptake of co-administered vaccine antigens into APC (14). Although MF59 has been shown to be safe and efficacious in a wide range of human clinical trials (15), its early clinical development yielded a number of important lessons. Initially, microemulsions were used as a delivery system for a synthetic immunopotentiator, a lipidated muramyl tripeptide (MTP-PE). MTP is a modified version of muramyl dipeptide (MDP), which was originally identified as the minimal adjuvant active structure isolated from the peptidoglycan of mycobacterial cell walls (16). Unlike LPS and its synthetic derivatives, which are also used as adjuvants (e.g., MPL), MDP does not activate TLR2 or TLR4, suggesting a different and as yet unidentified mechanism of action (17). However, MDP proved too toxic for use as an adjuvant and various synthetic derivatives were developed, including MTP$\mathrm{PE}$, which was lipidated to reduce toxicity and to allow easier incorporation into delivery vehicles (18). Unfortunately, microemulsion formulations of MTP-PE still showed an unacceptable level of reactogenicity, and were unsuitable for routine clinical use $(19,20)$. Nevertheless, these studies highlighted that MF59 microemulsion in combination with a recombinant envelope antigen from HIV was well-tolerated and had comparable immunogenicity to the formulation containing MTP-PE $(20,21)$. Therefore, MF59 was used in subsequent studies and proved sufficiently potent and safe to allow successful product development (13). Hence our early clinical experience with microemulsions served to illustrate the need for careful selection of the appropriate immunopotentiators to be included in adjuvant formulations.

The ability of MF59 to induce enhanced responses against influenza vaccine has been shown to be particularly advantageous when used in individuals with pre-existing chronic disease (22), or in a setting where there has been no preexposure to the circulating strain, including a potentially pandemic strain (23). Although MF59 has mainly been used in adults, it has also been shown to be safe and efficacious in neonates (24) and toddlers (25). Therefore, we believe that MF59 is appropriate for use in all age groups, including pe- diatric populations. MF59 has also shown enhanced potency over alum when used in non-human primates with protein polysaccharide conjugate vaccines (26). Nevertheless, although MF59 is a more potent adjuvant than alum (27), it cannot be expected to be suitable for all vaccines. MF59 works particularly well to enhance antibody and T cell proliferative responses $(27,28)$. However, it is generally poor for the induction of Th1 responses, which may be required to provide protective immunity against some intracellular pathogens. Nevertheless, Th1 immunopotentiators, including CpG have been successfully added to MF59 to improve its potency (29). Alternatively, MF59 can be used as a booster vaccine with recombinant proteins once a Th1 response has already been established by immunization with DNA (30) or attenuated viral vectors (31).

\section{Microparticles}

In the recent past, we have focused on an alternative delivery system for vaccines, comprising biodegradable microparticles prepared from the polymer, poly (lactide coglycolide) (PLG). Since PLG polymers have already been used for a variety of biomedical purposes, including the preparation of controlled release drug delivery systems, including human growth hormone (32), it was an excellent choice for a vaccine delivery system. Microparticles represent an attractive approach to vaccine delivery since it has been shown on many occasions that microparticles $(\sim 1 \mu \mathrm{m})$ are taken up efficiently by APC in vitro (33) and in vivo (34). In addition, microparticles have also been shown to be taken up by APC, which then migrated to the T-cell area of local lymph nodes and differentiated into DC (35).

Microparticles were first used as delivery systems for entrapped antigens in the early 1990 s by several groups $(36,37)$. In addition to antibody responses, early studies showed that microparticles were also able to induce cytotoxic $\mathrm{T}$ lymphocyte (CTL) responses in rodents $(38,39)$. This early work prompted speculation that microparticles may represent an attractive delivery system for the development of vaccines against tumors and intracellular pathogens (40). Nevertheless, the majority of initial work mainly focused on the use of microparticles for controlled release of entrapped antigens, with the objective of making single dose vaccines $(41,42)$. However, problems arose with this concept as a consequence of the well-documented degradation of antigens during encapsulation and release from PLG microparticles (43). Despite significant efforts in basic research over many years, microparticles with entrapped antigens have not yet moved forward into clinical trials as controlled release vaccines.

As a result of the problems associated with microencapsulation of vaccine antigens, we recently developed a novel approach of adsorbing antigens onto the surface of PLG microparticles. This approach avoids exposing antigens to damaging conditions during encapsulation and release. Importantly, we showed that the efficiency of antigen adsorption is enhanced by using charged surfactants during microparticle preparation (44). These novel microparticles were designed to perform as delivery systems to promote the uptake of adsorbed antigen into APC. Immunization with a recombinant antigen adsorbed to PLG microparticles resulted in the induction of potent antibody and T cell responses in mice (44) and in non-human primates (45). The adsorption of antigens 
onto the surface of microparticles results in the presentation of multiple copies of the antigen, which is similar to the surface of a pathogen. Recently, we showed that PLG microparticles induced potent antibody responses to adsorbed recombinant antigens from Neisseria meningitides type B (46).

The approach of adsorbing antigens onto the surface of charged microparticles has proven sufficiently flexible to allow the delivery of DNA vaccines. DNA vaccines have a number of potential advantages over alternative approaches, including the following; they are highly purified, several antigens can be easily included, they are inexpensive and they appear to be suitable for use in the presence of preexisting maternal immunity, which is certainly a problem for some existing live-attenuated vaccines e.g., measles. However, it is clear that the potency of DNA vaccines needs to be significantly improved in humans, since clinical trials have so far shown disappointing immune responses $(47,48)$. Small animal studies suggest that DNA vaccines are an effective means to prime the immune response in neonates (49) and the use of DNA vaccines to trigger a Th1 response could conceivably minimize the development of allergic diseases in children.

In a number of studies, we have shown that cationic PLG microparticles with adsorbed DNA vaccines induce significantly enhanced immune responses in comparison to immunization with naked DNA in mice (50) and non-human primates (51). Table VI shows a direct comparison between the immune responses, antibodies, CD4+ and CD8+ T-cell responses, following immunization of rhesus macaques with naked DNA encoding HIV gag and env antigens, or the same DNA adsorbed to PLG microparticles. In studies designed to determine the mechanism of action, we showed that cationic microparticles were able to deliver adsorbed DNA into DC, which were transfected, while naked DNA was unable to achieve this $(52,53)$. Previously, it had been reported that microparticles could be used as delivery systems for entrapped DNA vaccines (54). However, DNA is known to be damaged during encapsulation and release from PLG microparticles (55). In addition, it has never been shown that microencapsulation of DNA actually results in enhanced potency over naked DNA. Furthermore, DNA encapsulation efficiency is often low and release from microparticles can often be slow. In contrast, DNA strongly and efficiently adsorbs to cationic PLG microparticles, without damage, is rapidly released from the surface and results in significantly enhanced immune responses over naked DNA (50,56-58).

Cationic PLG microparticles can also be used as delivery systems for adsorbed adjuvants, including $\mathrm{CpG}$ oligonucleotides (59). In addition, microemulsions based on MF59 can be prepared with a charged surface, and these can also be used as delivery systems for DNA vaccines (60). The potential of a broad range of particulate vaccine delivery systems for new generation vaccines has recently been reviewed $(42,61,62)$.

\section{Delivery Systems for Mucosal Immunization}

Although the majority of vaccines have been administered by injection, mucosal immunization offers a number of important advantages; including easier administration, reduced adverse effects and the potential for frequent boosting. In addition, mucosal immunization has the potential to induce local immunity at the sites where pathogens often establish initial infection. Oral immunization would be particularly advantageous in isolated communities, where access to health care professionals is difficult. Moreover, mucosal immunization would avoid the problems of infection due to the re-use of needles, which is common in the developing world. However, the difficulty of developing mucosal and particularly oral vaccines using non-living approaches should not be underestimated. The oral vaccines that have been developed so far are mostly based on live attenuated organisms, which have the ability to infect and replicate within the intestinal epithelium. Protein, peptide, polysaccharide, and DNA antigens are extremely labile and will be degraded and damaged during passage through the gut, if not adequately protected. Consequently, intranasal immunization is an attractive approach, due to the absence of acidity and secreted enzymes in the nasal cavity, but also due to the easy access the nasal cavity offers with simple commercially available devices. Nevertheless, potent adjuvants and delivery systems will be required to enable the development of effective mucosal vaccines using non-living approaches. The potential of the intranasal approach has been highlighted recently by the successful licensure of a live-attenuated influenza vaccine, based on "coldadapted" strains, which replicate only in the colder temperature of the nasal cavity and not in the lungs.

The most potent mucosal adjuvants available are the bacterial toxins secreted from Escherichia coli and Vibrio cholerae, which are called heat-labile enterotoxin (LT) and cholera toxin $(\mathrm{CT})$. However, since these molecules are respectively responsible for travellers diarrhea and cholera, the "wild type" molecules are not appropriate for mucosal administration to humans. Therefore, these molecules have been manipulated to minimize toxicity, while retaining adjuvant activity (63). Encouragingly, nontoxic LT mutants have been shown to induce potent immune responses following mucosal immunization in several animal models (64). In addition, nontoxic LT mutants have also induced protective immunity, including protection against lethal pneumonia in neonatal mice (65). One particular mutant of LT, which was modified to eliminate the enzymatic and toxic activity in the A subunit (LTK63) (66), has recently been shown to be safe in a human clinical trial following intranasal administration. In previous studies, we showed that the potency of LTK63 for intranasal administration could be enhanced by co-administration with bioadhesive delivery systems $(67,68)$.

Table VI. Enhanced Immune Responses in Rhesus Macaques with PLG/DNA versus Naked DNA 2 Weeks After 2 Doses $(\mathrm{n}=5)$

\begin{tabular}{lccccc}
\hline \multicolumn{1}{c}{ Vaccine } & $\begin{array}{c}\text { Env titer } \\
(1-\mathrm{mg} \text { dose })\end{array}$ & $\begin{array}{c}\text { Gag titer } \\
(0.5-\mathrm{mg} \text { dose })\end{array}$ & $\begin{array}{c}\text { Gag CD4+ } \\
\text { (mean SI response) }\end{array}$ & $\begin{array}{c}\text { Gag CD4+ } \\
\text { (Animals with +ve responses) }\end{array}$ & $\begin{array}{c}\text { Gag CD8+ } \\
\text { (animals with +ve CTL responses) }\end{array}$ \\
\hline Naked DNA & 49 & 7 & 6.4 & $2 / 5$ & $1 / 5$ \\
PLG/DNA & 11,289 & 19,256 & 13.8 & $5 / 5$ & $3 / 5$ \\
Fold increase & 230 & 2750 & -2 & NA & NA \\
\hline
\end{tabular}


Figure 2 shows the ability of LTK63 and a bioadhesive delivery system (HYAFF microspheres) to induce significantly enhanced serum antibody responses in pigs (68). Encouragingly, recent data also suggests that LTK63 may prove sufficiently potent to allow the mucosal administration of pediatric combination vaccines (69).

In a recent study, we showed for the first time that IN immunization with a bacterial toxoid in humans could induce levels of serum antibody responses which correlated with protective immunity (70). In addition, the chitosan bioadhesive polymer delivery system also induced local mucosal immunity (70). However, this study involved the boosting of adults who were well primed as infants. It remains to be seen if IN immunization can induce effective priming immunization in humans. Recent data suggests that chitosan microparticles may also be used as an IN delivery system in conjunction with LTK63 (71).

Although oral delivery of nonliving vaccines remains challenging, some success has been achieved with nontoxic LT mutants (72,73). Hence, the potent immunogenicity and adjuvanticity of secreted bacterial toxins may be exploited to develop oral vaccines. The $\mathrm{B}$ subunit of cholera toxin has been used as a recombinant antigen in conjunction with killed whole bacterial cells to produce an effective oral vaccine against cholera (74), which was recently approved for use throughout Europe (Dukoral). In addition, a similar approach may also allow the development of a vaccine against E. coli (75). Nevertheless, there is a requirement for the development of optimal delivery systems if successful oral vaccines are to be produced for a wide range of organisms.

A range of microparticle-based delivery systems are available which can be designed to protect antigens against degradation in the gut, promote interaction with the epithe-

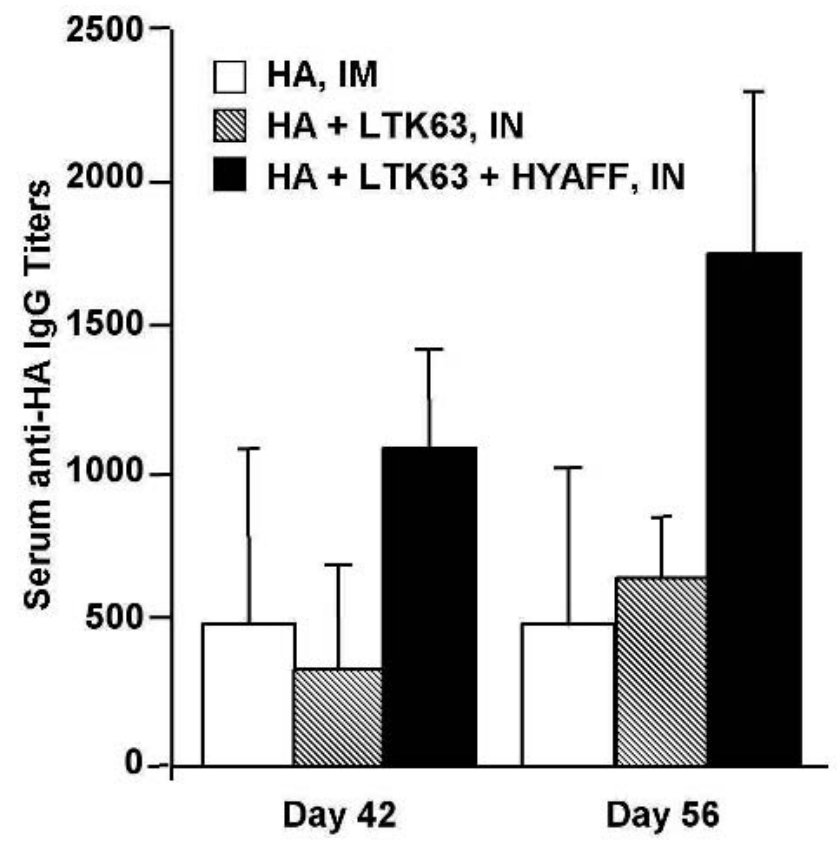

Fig. 2. Synergy between bioadhesive microspheres and an adjuvant for intranasal (IN) delivery of flu vaccine in pigs. The IN approach with both adjuvant (LTK63) and a bioadhesive delivery system (HYAFF microspheres) was significantly more potent than traditional intramuscular immunization (68). lium, or be taken up by the mucosal associated lymphoid tissues. Oral delivery systems with entrapped antigens have shown some success in clinical trials for allergy immunotherapy (76), and may offer promise as oral vaccine delivery systems (77). However, preliminary studies in small animal models did not suggest that these delivery systems alone would be sufficient to allow the development of non-living oral vaccines (78). In addition, although some success has been achieved in human clinical trials with vaccines entrapped in PLG microparticles, this approach would require significant improvements to enable the development of oral vaccines (79). Hence, it seems most likely that potent mucosal adjuvants will need to be combined with optimal delivery systems if oral vaccines are to be developed for nonreplicating vaccines. In experimental studies, oral delivery systems have also been used to enhance the potency of "vaccines" which downregulate the immune response, to induce "tolerance" to antigens associated with autoimmune disorders (80).

In addition to IN and oral immunization, vaccines may also be administered mucosally via aerosols, including measles vaccine $(81,82)$. Several million children have been immunized against measles in Mexico using the aerosol route, but concerns remain about the potential for adverse effects (83). In addition, studies are needed to determine the efficacy of combined measles-mumps-rubella vaccines when administered by aerosol, although studies with measles and rubella are promising (84). This approach might become more practical if dry powder inhalation devices can be developed, which are already used successfully for pulmonary drug delivery. Dry powder approaches could improve the stability of vaccines. The suitability of aerosol delivery for recombinant or other new generation vaccines has yet to be determined. In addition, developing suitable aerosol devices for widespread use in pediatric populations would present significant difficulties.

\section{Needle-Free Vaccine Delivery Devices}

In its broadest sense, the concept of "vaccine delivery" can be expanded to include a range of devices and physical delivery systems that are designed to allow immunization using noninvasive routes, not including mucosal delivery. Needle-free vaccination is attractive for a number of reasons. In the developed world, there are concerns about the number of vaccinations that are routinely administered to infants and toddlers, and it would be preferable if these could be administered without the use of needles. In addition, "needlephobia" is a significant problem that makes it difficult to convince adults to receive booster immunizations, even when the need is clear. Furthermore, new vaccines are likely to be introduced which will be targeted at adolescents, including some that will protect against sexually transmitted diseases. These vaccines would be easier to introduce if needle-free approaches were available. Moreover, the problem of needlephobia may become particularly acute if society needs to respond quickly to the emergence of a pandemic strain of influenza, or to the threat of a bioterrorism attack.

The problems related to needles are even greater in the developing world, where re-use of needles often results in the transmission of blood borne pathogens (e.g., HIV, HBV, and $\mathrm{HCV}$ ). Each year unsafe injection practice causes an estimated 22.5 million hepatitis B virus infections, 2.7 million 
HCV infections and 98,000 HIV infections. Hence, although vaccinations account for only a small number of the total injections administered, there is a clear need to improve the safety of immunization programs. In addition, accidental needle-stick injuries are also a significant problem in the developed world, leading to a significant number of infections amongst health care workers. One solution to this problem has been the development of single use "auto-disposable" (AD) syringes, which have permanently attached needles and plungers that lock in place once they are used. But AD syringes still represent an infectious threat that needs to be disposed safely, and they are more expensive than traditional needles and syringes (1). Therefore, there is strong interest in the development of needle-free devices for immunization, which could eliminate the transmission of infection due to needle re-use and needle-stick injuries. In addition, they could eliminate the risk of infections due to improper disposal of sharps. Because of these issues, the Global Alliance on Vaccines and Immunization (GAVI) has made the elimination of the use of needles and syringes a high priority.

\section{Needle-Free Approaches for Traditional Vaccines}

Multiple-use jet injector devices designed to deliver liquid vaccines through the skin were first developed in the 1950s and were widely used for mass immunization campaigns. However, these devices were constructed with reusable nozzles, which ultimately resulted in their downfall. It became apparent in the 1980s that these multidose devices were capable of transmitting blood-borne pathogens such as hepatitis B (85). These devices, which operated under high pressure gas, could become contaminated by blood "splashed back" from the patient. Therefore, multiple use jet injector devices are no longer recommended by the WHO and their use in the military has been terminated. More recently, single dose jet injector devices have been developed, which are capable of delivering liquid vaccines into the skin using high pressure (86). It was believed that jet injection devices would improve the immunogenicity of vaccines, due to better distribution in tissues, which should provide better access to APC than traditional needle immunization. However, superficial administration into and below the skin may also result in enhanced reactogenicity. One study compared two alternative jet injector devices with standard IM delivery for influenza vaccine and concluded that although the immune responses in all patients were comparable, the jet injector devices were associated with higher levels of pain and more local reactions (86). As an alternative, spring-powered liquid injection devices are also available and have been evaluated in the clinic. One study reported that five different vaccines were well-tolerated and had improved immunogenicity following delivery with a spring powered single use injection device (87). However, it was highlighted that the cost of the approach may prove limiting and that a detailed economic analysis was necessary prior to implementation for large scale immunization programs.

Although the majority of needle-free devices have delivered liquid vaccines, liquids are generally less stable than powders and are vulnerable to freezing. Therefore, needlefree dry powder vaccines are considered the optimal approach for large scale use, since these would be resistant to temperature fluctuations and should have greater stability. A helium driven single use dry powder injection device showed encouraging data in small animal models with an influenza vaccine (88) and was subsequently evaluated in non-human primates (89). However, the powdered influenza vaccine appeared inferior to standard IM immunization and an adjuvant was added to improve potency (89). Although the technical achievements with the Epidermal Powder Immunization (EPI) approach have been impressive, including its successful application to an alum adsorbed vaccine (90), the economics of the drying process used may make this approach difficult to apply to large scale vaccination. Unfortunately, it's difficult to see how this technology could become affordable for the developing world.

In summary, none of the needle-free devices available have yet attained broad acceptance in the medical community and the cost of some devices will make them difficult to implement for the developing world. One inherent problem with this approach relates to the desire of the device development companies to establish themselves as creators and marketers of proprietary systems. This results in a number of different devices and cartridges being available, which are not interchangeable. Therefore, if a company goes out of business their customers may find that a vaccine cartridge from a competing supplier cannot fill the gap. Clearly a degree of standardization in vaccine cartridges would provide benefit, but this can only be done once the optimal approach has been identified. An additional problem relates to the increased local reactogenicity with jet injectors in comparison to traditional injections, which are likely to limit the utilization of this approach.

\section{Needle-Free Approaches for DNA Vaccines}

High-pressure needle-free devices have also been used for the delivery of liquid DNA vaccines in clinical trials (91), as too have novel needle-free powder devices (92). Although the liquid based approach was associated with more adverse effects than traditional IM immunization, it was preferred by the trial participants (91). However, similar to previous studies with DNA vaccines, no antibody responses were detected. In contrast, delivery of a DNA vaccine directly into the epidermis coated onto gold beads ("gene gun") resulted in the induction of protective levels of antibodies against HBV, despite using very low doses of DNA (92). However, although HBV specific CD4+ and CD8+ responses were also induced, the levels of antibody responses induced by DNA compared unfavourably with the responses achieved following immunization with recombinant protein vaccines (93). Nevertheless, the DNA vaccine approach is mainly targeted at therapy in already infected individuals, a situation in which $\mathrm{T}$ cell responses might be expected to be more important. Interestingly, the type of immune response normally induced by DNA vaccines may be modified by direct delivery into the skin on gold beads. DNA typically induces a very polarized Th1 response following IM immunization, but this seems to change when DNA is administered using a gene gun (94). This observation could be explained by the low levels of $\mathrm{CpG}$ signals contained in the low doses of DNA administered by the gene gun. In recent studies, the potency of the gene gun has been enhanced by including LT and CT as genetic adjuvants (95). However, the inclusion of these biologically and enzymatically active molecules as adjuvants would result in a 
much more complex regulatory approval process. Hence, the gene gun is mostly being evaluated for the development of therapeutic vaccines against chronic infectious diseases and cancers. Overall, the suitability of the gene gun for widespread immunization is difficult to determine, since the potential role for DNA vaccines is not yet determined. However, the cost of the gene gun will most likely make it difficult to implement in the developing world. Moreover, a significant amount of safety data on DNA vaccines would be needed before the gene gun could ever be considered for pediatric populations.

\section{Topical Immunization onto the Skin}

An alternative needle-free approach to vaccine delivery, involves the use of microprojection arrays, designed to painlessly disrupt the outer layers of the skin to allow the vaccine access to epidermal langerhans cells. Microenhancer arrays were used to improve the potency of DNA vaccines in mice (96), while an alternative microprojection array was used for the delivery of a model protein vaccine in guinea pigs (97). While both approaches appear applicable to a wide variety of vaccines, their suitability for human vaccination remains to be determined.

Perhaps the most attractive needle-free approach to vaccine delivery currently being explored involves transcutaneous immunization, through topical application of vaccine patches (98). This approach has recently provided encouraging observations in clinical trials involving a potential travelers diarrhea vaccine $(99,100)$. These studies showed the need for an LT adjuvant to be included in the vaccine, although a number of alternative adjuvants were also effective in preclinical studies (101). The ability of LT mutants to perform as adjuvants for topical application may eliminate some safety concerns associated with the use of wild type toxins $(101,102)$. An interesting recent development is the use of a topically applied "immunostimulatory patch" to enhance the immunogenicity of a locally injected influenza vaccine in humans (103). Although this approach is promising, the technology would need to undergo significant improvements to allow topical application of existing vaccines to pediatric populations. Perhaps this technology has more potential for the administration of booster doses to a well-primed adult population, following primary immunization using a more traditional route.

\section{CONCLUSIONS}

In the past decade there have been a number of significant advances in technologies to identify, express and deliver vaccine antigens. As a consequence, many of the vaccine candidates currently under evaluation look very different from traditional vaccines. In particular, there has been a shift away from the use of whole pathogens or inactivated subunits, toward the use of recombinant purified proteins. This has resulted in the need to develop novel adjuvants and delivery systems to improve the immunogenicity of these antigens. Optimal new generation vaccines, particularly from a safety perspective, will contain recombinant protein antigens, purified synthetic immunopotentiators, which represent welldefined PAMPs, and a delivery system designed to ensure that both the antigen and the adjuvant are targeted efficiently to APC (Fig. 1). Formulation of the vaccine into a delivery system will fulfill two main purposes: (i) enhance potency by focusing the effects of the immunopotentiator onto the key cells of the immune system and (ii) limit adverse effects by minimizing the systemic distribution of the immunopotentiator. It is clear that novel adjuvant and delivery technologies will be required to enable the successful development of vaccines against diseases that have not yielded to traditional approaches.

In the recent past, there have been advances in needlefree technologies for immunization. Needle-free vaccination includes a variety of approaches for mucosal immunization, and patches and devices allowing delivery of vaccines through or into the skin. Although some mucosal vaccines are commercially available, needle-free approaches involving devices have so far failed to achieve broad acceptance, despite their potential advantages. There are a number of reasons to explain the current lack of commercial success in this area, but safety concerns are significant, as too are concerns about the potential cost of novel devices. In addition, there are concerns that the efficacy of existing vaccines might be impaired if they were to be administered by alternative routes, or using novel devices. As a consequence of the high level of awareness of the role of vaccines in protecting children against infectious disease, infant immunization is routinely undertaken in all developed societies. Therefore, while new vaccines may be added to the list of those already recommended, introducing needle-free delivery approaches for existing vaccines will be exceptionally difficult. In addition to the significant hurdle of proving that the novel approach is safe, it will also be necessary to undertake studies to prove that the new technology does not negatively impact the potency of the vaccine. A clear demonstration of noninferiority for the novel approach will be required, including a large comparison with the existing approach. It's conceivable that the requirement for demonstration of noninferiority could even be extended to include vaccines that are administered by the traditional route at similar times to the novel approach. Hence it is clear that for existing vaccines, the greatest opportunity for the introduction of a novel delivery technology is represented by their use as booster products for older children, adolescents or adults. In this situation, the novel delivery approach would not need to show that it primes as well as the existing approach, rather it would need to show that it can provide an effective boost in well-primed individuals. In addition, the novel technology would be administered to a mature and cooperative population, rather than infants or young children.

Hence, it is clear that needle-free vaccine delivery technologies will prove most easy to apply for novel vaccines, particularly if efficacy is dependent on a novel route of delivery. This may particularly apply to a number of sexually transmitted diseases, including HIV, Chlamydia trachomatis, herpes simplex or gonorrhea, or could equally apply to diseases of the respiratory tract, including respiratory syncytial virus, parainfluenza virus or SARS. Alternatively, novel needle-free delivery approaches may become established to improve patient compliance for annual vaccination (e.g., influenza) or to protect against a potential bio-terrorist attack, or an emerging pandemic strain of influenza. In addition, novel delivery may achieve market success through improving the convenience of immunization or due to minimizing pain.

Although a number of needle-free technologies have 
shown significant promise, the vaccine industry continues to adopt very conservative approaches. This is mainly due to concerns about the potential for adverse effects with new technologies. Vaccination policy continues to be subject to a high level of scrutiny, with a significant emphasis on safety and very little tolerance for adverse effects, even when relatively minor in relation to the benefit. In addition, vaccination bears a heavy responsibility in ensuring protection for the annual birth cohort against a number of important infectious diseases. Hence a conservative approach is appropriate, but results in significant resistance to change, even when the potential advantages are clear. Nevertheless, figures show that the re-use of injection equipment in 2000 respectively accounted for $32 \%, 40 \%$, and $5 \%$ of new HBV, HCV, and HIV infections (104). These figures highlight the need for a change in current practice. The continued support of national and international agencies is crucial to the development of novel vaccine delivery technologies that have the potential to improve the safety of vaccination practice throughout the world.

New adjuvants and delivery systems may allow vaccines to treat chronic infections such as HIV and HCV, or to treat a variety of residual tumors remaining after surgery. In addition, novel approaches may also be required to treat autoimmune disorders. Novel devices and routes of delivery may have a particularly important role to play in situations where chronic administration of vaccine products is required, as might be envisaged for therapy, and ease of administration may become crucial to enable effective patient compliance in these situations.

\section{ACKNOWLEDGMENTS}

We would like to acknowledge the contributions of our colleagues in Chiron for their research efforts and ideas. Also, we would like to acknowledge Nelle Cronen for manuscript formatting.

\section{REFERENCES}

1. L. Jodar, P. Duclos, J. B. Milstien, E. Griffiths, M. T. Aguado, and C. J. Clements. Ensuring vaccine safety in immunization programmes-a WHO perspective. Vaccine 19:1594-1605 (2001).

2. N. M. Valiante, D. O'Hagan. and J. Ulmer. Innate Immunity and biodefence vaccines. Cell. Microbiol. 5:755-760 (2003).

3. J. M. Polo and T. W. Dubensky. Virus-based vectors for human vaccine applications. Drug Discov. Today 7:719-727 (2002).

4. G. Ramon. Procedures pour accroitre la production des antitoxines. Annales de l'Institut Pasteur 40:1-10 (1926).

5. R. Medzhitov and C. A. Janeway Jr. An ancient system of host defense. Curr. Opin. Immunol. 10:12-15 (1998).

6. A. Bendelac and R. Medzhitov. Adjuvants of immunity: harnessing innate immunity to promote adaptive immunity. J. Exp. Med. 195:F19-F23 (2002).

7. R. Medzhitov. Toll-like receptors and innate immunity. Nat. Rev. Immunol. 1:135-145 (2001).

8. D. T. O'Hagan and N. M. Valiante. Recent advances in the discovery and delivery of vaccines and adjuvants. Nat. Rev. Drug Discov. 2:727-735 (2003).

9. F. R. Vogel and M. F. Powell. A compendium of vaccine adjuvants and excipients. In M. F. Powelland M. J. Newman (eds), Vaccine Design: The Subunit and Adjuvant Approach, Plenum Press, New York, 1995, pp. 141-228.

10. R. M. Zinkernagel, S. Ehl, P. Aichele, S. Oehen, T. Kundig, and $\mathrm{H}$. Hengartner. Antigen localisation regulates immune responses in a dose- and time-dependent fashion: a geographical view of immune reactivity. Immunol. Rev. 156:199-209 (1997).

11. R. Edelman. Adjuvants for the future. In M. M. Levine, G. C.
Woodrow, J. B. Kaper, and G. S. Cobon (eds), New Generation Vaccines, Vol. Second, Marcel Dekker, Inc., New York, 1997, pp. 173-192.

12. R. T. Kenney and R. Edelman. Survey of human-use adjuvants. Expert Review Vaccines 2:167-188 (2003).

13. G. Ott. The Adjuvant MF59: A ten year perspective. In D. O'Hagan (ed), Vaccine Adjuvants; Preparation methods and research protocols (D. O'Hagan, ed), Humana Press, 2001, pp. 211-228.

14. M. Dupuis, K. Denis-Mize, A. LaBarbara, W. Peters, I. F. Charo, D. M. McDonald, and G. Ott. Immunization with the adjuvant MF59 induces macrophage trafficking and apoptosis. Eur. J. Immunol. 31:2910-2918 (2001).

15. A. Podda and G. Del Giudice. MF59-adjuvanted vaccines: increased immunogenicity with an optimal safety profile. Expert Rev Vaccines 2:197-203 (2003).

16. F. Ellouz, A. Adam, R. Ciorbaru, and E. Lederer. Minimal structural requirements for adjuvant activity of bacterial peptidoglycan derivatives. Biochem. Biophys. Res. Commun. 59: 1317-1325 (1974).

17. V. F. Vidal, N. Casteran, C. J. Riendeau, H. Kornfeld, E. C. Darcissac, A. Capron, and G. M. Bahr. Macrophage stimulation with Murabutide, an HIV-suppressive muramyl peptide derivative, selectively activates extracellular signal-regulated kinases 1 and 2, C/EBPbeta and STAT1: role of CD14 and Toll-like receptors 2 and 4. Eur. J. Immunol. 31:1962-1971 (2001).

18. J. Wintsch, C. L. Chaignat, D. G. Braun, M. Jeannet, H. Stalder, S. Abrignani, D. Montagna, F. Clavijo, P. Moret, J. M. Dayer, et al. Safety and immunogenicity of a genetically engineered human immunodeficiency virus vaccine. J. Infect. Dis. 163:219-225 (1991).

19. W. Keitel, R. Couch, N. Bond, S. Adair, G. Van Nest, and C. Dekker. Pilot evaluation of influenza virus vaccine (IVV) combined with adjuvant. Vaccine 11:909-913 (1993).

20. M. C. Keefer, B. S. Graham, M. J. McElrath, T. J. Matthews, D. M. Stablein, L. Corey, P. F. Wright, D. Lawrence, P. E. Fast, K Weinhold, R. H. Hsieh, D. Chernoff, C. Dekker, and R. Dolin. Safety and immunogenicity of Env 2-3, a human immunodeficiency virus type 1 candidate vaccine, in combination with a novel adjuvant, MTP-PE/MF59. NIAID AIDS Vaccine Evaluation Group. AIDS Res. Hum. Retroviruses 12:683-693 (1996).

21. J. O. Kahn, F. Sinangil, J. Baenziger, N. Murcar, D. Wynne, R. L. Coleman, K. S. Steimer, C. L. Dekker, and D. Chernoff. Clinical and immunologic responses to human immunodeficiency virus (HIV) type 1SF2 gp120 subunit vaccine combined with MF59 adjuvant with or without muramyl tripeptide dipalmitoyl phosphatidylethanolamine in non-HIV-infected human volunteers. J. Infect. Dis. 170:1288-1291 (1994).

22. A. Banzhoff, P. Nacci, and A. Podda. A new MF59-adjuvanted influenza vaccine enhances the immune response in the elderly with chronic diseases: results from an immunogenicity metaanalysis. Gerontology 49:177-184 (2003).

23. K. G. Nicholson, A. E. Colegate, A. Podda, I. Stephenson, J. Wood, E. Ypma, and M. C. Zambon. Safety and antigenicity of non-adjuvanted and MF59-adjuvanted influenza A/Duck/ Singapore/97 (H5N3) vaccine: a randomised trial of two potential vaccines against H5N1 influenza. Lancet 357:1937-1943 (2001).

24. C. K. Cunningham, D. W. Wara, M. Kang, T. Fenton, E. Hawkins, J. McNamara, L. Mofenson, A. M. Duliege, D. Francis, E. J. McFarland, and W. Borkowsky. Safety of 2 recombinant human immunodeficiency virus type 1 (hiv-1) envelope vaccines in neonates born to hiv-1-infected women. Clin. Infect. Dis. 32:801-807 (2001).

25. D. K. Mitchell, S. J. Holmes, R. L. Burke, A. M. Duliege, and S. P. Adler. Immunogenicity of a recombinant human cytomegalovirus gB vaccine in seronegative toddlers. Pediatr. Infect. Dis. J. 21:133-138 (2002).

26. D. M. Granoff, Y. E. McHugh, H. V. Raff, A. S. Mokatrin, and G. A. Van Nest. MF59 adjuvant enhances antibody responses of infant baboons immunized with Haemophilus influenzae type $b$ and Neisseria meningitidis group C oligosaccharide-CRM197 conjugate vaccine. Infect. Immun. 65:1710-1715 (1997). 
27. G. Ott. Vaccine Design: The Subunit and Adjuvant Approach, Plenum Press, New York, 1995.

28. G. Ott, R. Radhakrishnan, J.-H. Fang, and M. Hora. The adjuvant MF59: a 10-year perspective. In D. T. O'Hagan (ed), Vaccine Adjuvants: Preparation Methods and Research Protocols (D. T. O'Hagan, ed), Humana Press Inc., Totowa, NJ, 2000, pp. 211-228.

29. D. T. O’Hagan, M. Singh, J. Kazzaz, M. Ugozzoli, M. Briones, J. Donnelly, and G. Ott. Synergistic adjuvant activity of immunostimulatory DNA and oil/water emulsions for immunization with HIV p55 gag antigen. Vaccine 20:3389-3398 (2002).

30. S. Cherpelis, I. Shrivastava, A. Gettie, X. Jin, D. D. Ho, S. W. Barnett, and L. Stamatatos. DNA vaccination with the human immunodeficiency virus type 1 SF162DeltaV2 envelope elicits immune responses that offer partial protection from simian/ human immunodeficiency virus infection to $\mathrm{CD} 8(+) \mathrm{T}$-celldepleted rhesus macaques. J. Virol. 75:1547-1550 (2001).

31. A. V. E. G. P. T.-. AIDS. Cellular and humoral immune responses to a canarypox vaccine containing human immunodeficiency virus type 1 Env, Gag, and Pro in combination with rgp120. J. Infect. Dis. 183:563-570 (2001).

32. O. L. Johnson, J. L. Cleland, H. J. Lee, M. Charnis, E. Duenas, W. Jaworowicz, D. Shepard, A. Shahzamani, A. J. Jones, and S. D. Putney. A month-long effect from a single injection of microencapsulated human growth hormone. Nat. Med. 2:795-799 (1996).

33. Y. Tabata and Y. Ikada. Macrophage phagocytosis of biodegradable microspheres composed of L-lactic acid/glycolic acid homo- and copolymers. J. Biomed. Mater. Res. 22:837-858 (1988).

34. K. D. Newman, P. Elamanchili, G. S. Kwon and J. Samuel. Uptake of poly(D,L-lactic-co-glycolic acid) microspheres by antigen-presenting cells in vivo. J. Biomed. Mater. Res. 60:480-486 (2002).

35. G. J. Randolph, K. Inaba, D. F. Robbiani, R. M. Steinman, and W. A. Muller. Differentiation of phagocytic monocytes into lymph node dendritic cells in vivo. Immunity 11:753-761 (1999).

36. J. H. Eldridge, J. K. Staas, J. A. Meulbroek, T. R. Tice, and R. M. Gilley. Biodegradable and biocompatible poly(DL-lactideco-glycolide) microspheres as an adjuvant for staphylococcal enterotoxin B toxoid which enhances the level of toxin-neutralizing antibodies. Infect. Immun. 59:2978-2986 (1991).

37. D. T. O’Hagan, D. Rahman, J. P. McGee, H. Jeffery, M. C. Davies, P. Williams, S. S. Davis, and S. J. Challacombe. Biodegradable microparticles as controlled release antigen delivery systems. Immunology 73:239-242 (1991).

38. K. J. Maloy, A. M. Donachie, D. T. O'Hagan, and A. M. Mowat. Induction of mucosal and systemic immune responses by immunization with ovalbumin entrapped in poly(lactide-co-glycolide) microparticles. Immunology 81:661-667 (1994).

39. A. Moore, P. McGuirk, S. Adams, W. C. Jones, J. P. McGee, D. T. O'Hagan, and K. H. Mills. Immunization with a soluble recombinant HIV protein entrapped in biodegradable microparticles induces HIV-specific CD8+ cytotoxic T lymphocytes and CD4+ Th1 cells. Vaccine 13:1741-1749 (1995).

40. L. D. Falo, M. Kovacsovicsbankowski, K. Thompson, and K. L. Rock. Targeting antigen into the phagocytic pathway in-vivo induces protective tumor-immunity. Nat. Med. 1:649-653 (1995).

41. D. T. O'Hagan. Microparticles and polymers for the mucosal delivery of vaccines. Adv. Drug Deliv. Rev. 34:305-320 (1998).

42. D. O'Hagan and M. Singh. Microparticles as vaccine adjuvants and delivery systems. Expert Review Vaccines 2:269-283 (2003).

43. S. P. Schwendeman. Recent advances in the stabilization of proteins encapsulated in injectable PLGA delivery systems. Crit. Rev. Ther. Drug Carrier Syst. 19:73-98 (2002).

44. J. Kazzaz, J. Neidleman, M. Singh, G. Ott, and D. T. O'Hagan. Novel anionic microparticles are a potent adjuvant for the induction of cytotoxic T lymphocytes against recombinant p55 gag from HIV-1. J. Control. Release 67:347-356 (2000).

45. G. R. Otten, M. Schaefer, C. Greer, M. Calderon-Cacia, D. Coit, J. Kazzaz, A. Medina-Selby, M. Selby, M. Singh, M. Ugozzoli, J. zur Megede, S. Barnett, D. O'Hagan, J. Donnelly, and J. B. Ulmer. Induction of broad and potent anti-HIV immune responses in rhesus macaques by priming with a DNA vaccine and boosting with protein-adsorbed PLG microparticles. J. Virol. 77:6087-6092 (2003).

46. M. Singh, J. Kazzaz, J. Chesko, E. Soenawan, M. Ugozzoli, M. M. Giuliani, M. Pizza, R. Rappuoli, and D. O’Hagan. Anionic microparticles are a potent delivery system for recombinant antigens from neisseria meningitidis serotype B. J. Pharm. Sci. 93: 273-282 (2004).

47. R. Wang, D. L. Doolan, T. P. Le, R. C. Hedstrom, K. M. Coonan, Y. Charoenvit, T. R. Jones, P. Hobart, M. Margalith, J. Ng, W. R. Weiss, M. Sedegah, C. de Taisne, J. A. Norman, and S. L. Hoffman. Induction of antigen-specific cytotoxic T lymphocytes in humans by a malaria DNA vaccine. Science 282:476480 (1998).

48. T. P. Le, K. M. Coonan, R. C. Hedstrom, Y. Charoenvit, M. Sedegah, J. E. Epstein, S. Kumar, R. Wang, D. L. Doolan, J. D. Maguire, S. E. Parker, P. Hobart, J. Norman, and S. L. Hoffman. Safety, tolerability and humoral immune responses after intramuscular administration of a malaria DNA vaccine to healthy adult volunteers. Vaccine 18:1893-1901 (2000).

49. X. Martinez, C. Brandt, F. Saddallah, C. Tougne, C. Barrios, F. Wild, G. Dougan, P. H. Lambert, and C. A. Siegrist. DNA immunization circumvents deficient induction of $t$ helper type 1 and cytotoxic t lymphocyte responses in neonates and during early life. Proc.s Natl. Acad. Sci. USA 94:8726-8731 (1997).

50. M. Singh, M. Briones, G. Ott, and D. O'Hagan. Cationic microparticles: A potent delivery system for DNA vaccines. Proc. Natl. Acad. Sci. USA 97:811-816 (2000).

51. D. O’Hagan, M. Singh, M. Ugozzoli, C. Wild, S. Barnett, M. Chen, M. Schaefer, B. Doe, G. R. Otten, and J. B. Ulmer. Induction of potent immune responses by cationic microparticles with adsorbed human immunodeficiency virus DNA vaccines. $J$. Virol. 75:9037-9043 (2001).

52. K. S. Denis-Mize, M. Dupuis, M. L. MacKichan, M. Singh, B. Doe, D. O'Hagan, J. B. Ulmer, J. J. Donnelly, D. M. McDonald, and G. Ott. Plasmid DNA adsorbed onto cationic microparticles mediates target gene expression and antigen presentation by dendritic cells. Gene Ther. 7:2105-2112 (2000).

53. K. Denis-Mize, M. Dupuis, M. Singh, C. Woo, M. Ugozzoli, D. O'Hagan, J. Donnelly, G. Ott, and D. M. McDonald. Mechanisms of Increased Immunogenicity for DNA-Based Vaccines Absorbed onto Cationic Microparticles. Cell. Immunol. 225:1220 (2003).

54. M. L. Hedley, J. Curley, and R. Urban. Microspheres containing plasmid-encoded antigens elicit cytotoxic T-cell responses. Nat. Med. 4:365-368 (1998).

55. S. Ando, D. Putnam, D. W. Pack, and R. Langer. PLGA microspheres containing plasmid DNA: preservation of supercoiled DNA via cryopreparation and carbohydrate stabilization. $J$. Pharm. Sci. 88:126-130 (1999).

56. D. O'Hagan, M. Singh, M. Ugozzoli, C. Wild, S. Barnett, M. Chen, G. R. Otten, and J. B. Ulmer. Induction of potent immune responses by cationic microparticles with adsorbed HIV DNA vaccines. J. Virol. 75:9037-9043 (2001).

57. M. Briones, M. Singh, M. Ugozzoli, J. Kazzaz, S. Klakamp, G. Ott, and D. O'Hagan. The preparation, characterization, and evaluation of cationic microparticles for DNA vaccine delivery. Pharm. Res. 18:709-711 (2001).

58. M. Singh, M. Ugozzoli, M. Briones, J. Kazzaz, E. Soenawan, and D. O'Hagan. The effect of CTAB concentration in cationic PLG microparticles on DNA adsorption and in vivo performance. Pharm. Res. 20:244-248 (2003).

59. M. Singh, G. Ott, J. Kazzaz, M. Ugozzoli, M. Briones, J. Donnelly, and D. T. O'Hagan. Cationic microparticles are an effective delivery system for immune stimulatory CpG DNA. Pharm. Res. 18:1476-1479 (2001).

60. G. Ott, M. Singh, J. Kazzaz, M. Briones, E. Soenawan, M. Ugozzoli, and D. T. O'Hagan. A cationic sub-micron emulsion (MF59/DOTAP) is an effective delivery system for DNA vaccines. J. Control. Release 79:1-5 (2002).

61. S. M. Michalek, D. T. O'Hagan, S. Gould-Fogerite, G. F. Rimmelzwaan, and A. D. M. E. Osterhaus. Antigen delivery systems: nonliving microparticles, liposomes, cochleates, and ISCOMS. In P. L. Ogra, J. Mestecky, M. E. Lamm, W. Strober, J. Bienenstrock, and J. R. McGhee (eds), Mucosal Immunology, Academic Press, San Diego, 1999, pp. 759-778. 
62. M. Singh and D. T. O'Hagan. Recent advances in vaccine adjuvants. Pharm. Res. 19:715-728 (2002).

63. R. Rappuoli, M. Pizza, G. Douce, and G. Dougan. Structure and mucosal adjuvanticity of cholera and Escherichia coli heat-labile enterotoxins. Immunol. Today 20:493-500 (1999).

64. M. Pizza, M. M. Giuliani, M. R. Fontana, E. Monaci, G. Douce, G. Dougan, K. H. Mills, R. Rappuoli, and G. Del Giudice. Mucosal vaccines: non toxic derivatives of LT and CT as mucosal adjuvants. Vaccine 19:2534-2541 (2001).

65. H. Jakobsen, S. Bjarnarson, G. Del Giudice, M. Moreau, C. A. Siegrist, and I. Jonsdottir. Intranasal immunization with pneumococcal conjugate vaccines with LT-K63, a nontoxic mutant of heat-Labile enterotoxin, as adjuvant rapidly induces protective immunity against lethal pneumococcal infections in neonatal mice. Infect. Immun. 70:1443-1452 (2002).

66. S. Peppoloni, P. Ruggiero, M. Contorni, M. Morandi, M. Pizza, R. Rappuoli, A. Podda, and G. Del Giudice. Mutants of the Escherichia coli heat-labile enterotoxin as safe and strong adjuvants for intranasal delivery of vaccines. Expert Review Vaccines 2:285-293 (2003).

67. B. C. Baudner, O. Balland, M. M. Giuliani, P. Von Hoegen, R. Rappuoli, D. Betbeder, and G. Del Giudice. Enhancement of protective efficacy following intranasal immunization with vaccine plus a nontoxic LTK63 mutant delivered with nanoparticles. Infect. Immun. 70:4785-4790 (2002).

68. M. Singh, M. Briones, and D. T. O'Hagan. A novel bioadhesive intranasal delivery system for inactivated influenza vaccines. $J$. Control. Release 70:267-276 (2001).

69. M. Ugozzoli, G. Santos, J. Donnelly, and D. T. O’Hagan. Potency of a genetically detoxified mucosal adjuvant derived from the heat-labile enterotoxin of Escherichia coli (LTK63) is not adversely affected by the presence of preexisting immunity to the adjuvant. J. Infect. Dis. 183:351-354 (2001).

70. K. H. Mills, C. Cosgrove, E. A. McNeela, A. Sexton, R. Giemza, I. Jabbal-Gill, A. Church, W. Lin, L. Illum, A. Podda, R. Rappuoli, M. Pizza, G. E. Griffin, and D. J. Lewis. Protective levels of diphtheria-neutralizing antibody induced in healthy volunteers by unilateral priming-boosting intranasal immunization associated with restricted ipsilateral mucosal secretory immunoglobulin a. Infect. Immun. 71:726-732 (2003).

71. B. C. Baudner, M. M. Giuliani, J. C. Verhoef, R. Rappuoli, H. E. Junginger, and G. D. Giudice. The concomitant use of the LTK63 mucosal adjuvant and of chitosan-based delivery system enhances the immunogenicity and efficacy of intranasally administered vaccines. Vaccine 21:3837-3844 (2003).

72. G. Douce, V. Giannelli, M. Pizza, D. Lewis, P. Everest, R. Rappuoli, and G. Dougan. Genetically detoxified mutants of heatlabile toxin from Escherichia coli are able to act as oral adjuvants. Infect. Immun. 67:4400-4406 (1999).

73. J. D. Barackman, G. Ott, S. Pine, and D. T. O'Hagan. Oral administration of influenza vaccine in combination with the adjuvants LT-K63 and LT-R72 induces potent immune responses comparable to or stronger than traditional intramuscular immunization. Clin. Diagn. Lab. Immunol. 8:652-657 (2001).

74. J. D. Clemens, D. A. Sack, J. R. Harris, F. Van Loon, J. Chakraborty, F. Ahmed, M. R. Rao, M. R. Khan, M. Yunus, N. Huda, et al. Field trial of oral cholera vaccines in Bangladesh: results from three-year follow-up. Lancet 335:270-273 (1990).

75. M. Jertborn, C. Ahren, J. Holmgren, and A. M. Svennerholm. Safety and immunogenicity of an oral inactivated enterotoxigenic Escherichia coli vaccine. Vaccine 16:255-260 (1998).

76. M. A. Van Deusen, B. L. Angelini, K. M. Cordoro, B. A. Seiler, L. Wood, and D. P. Skoner. Efficacy and safety of oral immunotherapy with short ragweed extract. Ann. Allergy Asthma Immunol. 78:573-580 (1997).

77. D. O'Hagan. Microparticles and polymers for the mucosal delivery of vaccines. Adv. Drug Deliv. Rev. 34:305-320 (1998).

78. S. L. Jain, K. S. Barone, M. P. Flanagan, and J. G. Michael. Activation patterns of murine B cells after oral administration of an encapsulated soluble antigen. Vaccine 14:1291-1297 (1996).

79. D. E. Katz, A. J. DeLorimier, M. K. Wolf, E. R. Hall, F. J. Cassels, J. E. van Hamont, R. L. Newcomer, M. A. Davachi, D. N. Taylor, and C. E. McQueen. Oral immunization of adult volunteers with microencapsulated enterotoxigenic Escherichia coli (ETEC) CS6 antigen. Vaccine 21:341-346 (2003).

80. J. Holmgren, C. Czerkinsky, K. Eriksson, and A. Mharandi. Mucosal immunisation and adjuvants: a brief overview of recent advances and challenges. Vaccine 21:S89-S95 (2003).

81. A. B. Sabin, A. Flores Arechiga, J. Fernandez de Castro, J. L. Sever, D. L. Madden, I. Shekarchi, and P. Albrecht. Successful immunization of children with and without maternal antibody by aerosolized measles vaccine. I. Different results with undiluted human diploid cell and chick embryo fibroblast vaccines. JAMA 249:2651-2662 (1983).

82. A. Dilraj, F. T. Cutts, J. F. de Castro, J. G. Wheeler, D. Brown, C. Roth, H. M. Coovadia, and J. V. Bennett. Response to different measles vaccine strains given by aerosol and subcutaneous routes to schoolchildren: a randomised trial. Lancet $\mathbf{3 5 5}$ : 798-803 (2000).

83. F. T. Cutts, A. Henao-Restrepo, and J. M. Olive. Measles elimination: progress and challenges. Vaccine 17:S47-S52 (1999).

84. J. Sepulveda-Amor, J. L. Valdespino-Gomez, L. Garcia-Garcia Mde, J. Bennett, R. Islas-Romero, G. Echaniz-Aviles, and J. F. de Castro. A randomized trial demonstrating successful boosting responses following simultaneous aerosols of measles and rubella (MR) vaccines in school age children. Vaccine 20:27902795 (2002).

85. J. Canter, K. Mackey, L. S. Good, R. R. Roberto, J. Chin, W. W. Bond, M. J. Alter, and J. M. Horan. An outbreak of hepatitis B associated with jet injections in a weight reduction clinic. Arch. Intern. Med. 150:1923-1927 (1990).

86. L. A. Jackson, G. Austin, R. T. Chen, R. Stout, F. DeStefano, G. J. Gorse, F. K. Newman, O. Yu, and B. G. Weniger. Safety and immunogenicity of varying dosages of trivalent inactivated influenza vaccine administered by needle-free jet injectors. Vaccine 19:4703-4709 (2001).

87. I. Parent du Chatelet, J. Lang, M. Schlumberger, E. Vidor, G. Soula, A. Genet, S. M. Standaert, and P. Saliou. Clinical immunogenicity and tolerance studies of liquid vaccines delivered by jet-injector and a new single-use cartridge (Imule): comparison with standard syringe injection. Imule Investigators Group. Vaccine 15:449-458 (1997).

88. D. Chen, R. L. Endres, C. A. Erickson, K. F. Weis, M. W. McGregor, Y. Kawaoka, and L. G. Payne. Epidermal immunization by a needle-free powder delivery technology: immunogenicity of influenza vaccine and protection in mice. Nat. Med. 6:1187-1190 (2000).

89. D. Chen, R. Endres, Y. F. Maa, C. R. Kensil, P. WhitakerDowling, A. Trichel, J. S. Youngner, and L. G. Payne. Epidermal powder immunization of mice and monkeys with an influenza vaccine. Vaccine 21:2830-2836 (2003).

90. Y. F. Maa, L. Zhao, L. G. Payne, and D. Chen. Stabilization of alum-adjuvanted vaccine dry powder formulations: mechanism and application. J. Pharm. Sci. 92:319-332 (2003).

91. J. E. Epstein, E. J. Gorak, Y. Charoenvit, R. Wang, N. Freydberg, O. Osinowo, T. L. Richie, E. L. Stoltz, F. Trespalacios, J. Nerges, J. Ng, V. Fallarme-Majam, E. Abot, L. Goh, S. Parker, S. Kumar, R. C. Hedstrom, J. Norman, R. Stout, and S. L. Hoffman. Safety, tolerability, and lack of antibody responses after administration of a PfCSP DNA malaria vaccine via needle or needle-free jet injection, and comparison of intramuscular and combination intramuscular/intradermal routes. Hum. Gene Ther. 13:1551-1560 (2002).

92. M. J. Roy, M. S. Wu, L. J. Barr, J. T. Fuller, L. G. Tussey, S. Speller, J. Culp, J. K. Burkholder, W. F. Swain, R. M. Dixon, G. Widera, R. Vessey, A. King, G. Ogg, A. Gallimore, J. R. Haynes, and D. Heydenburg Fuller. Induction of antigenspecific CD8+ T cells, T helper cells, and protective levels of antibody in humans by particle-mediated administration of a hepatitis B virus DNA vaccine. Vaccine 19:764-778 (2000).

93. T. C. Heineman, M. L. Clements-Mann, G. A. Poland, R. M. Jacobson, A. E. Izu, D. Sakamoto, J. Eiden, G. A. Van Nest, and H. H. Hsu. A randomized, controlled study in adults of the immunogenicity of a novel hepatitis B vaccine containing MF59 adjuvant. Vaccine 17:2769-2778 (1999).

94. M. J. McCluskie, C. L. Brazolot Millan, R. A. Gramzinski, H. L. Robinson, J. C. Santoro, J. T. Fuller, G. Widera, J. R. Haynes, 
R. H. Purcell, and H. L. Davis. Route and method of delivery of DNA vaccine influence immune responses in mice and nonhuman primates. Mol. Med. 5:287-300 (1999).

95. J. Arrington, R. P. Braun, L. Dong, D. H. Fuller, M. D. Macklin, S. W. Umlauf, S. J. Wagner, M. S. Wu, L. G. Payne, and J. R. Haynes. Plasmid vectors encoding cholera toxin or the heatlabile enterotoxin from Escherichia coli are strong adjuvants for DNA vaccines. J. Virol. 76:4536-4546 (2002).

96. J. A. Mikszta, J. B. Alarcon, J. M. Brittingham, D. E. Sutter, R. J. Pettis, and N. G. Harvey. Improved genetic immunization via micromechanical disruption of skin-barrier function and targeted epidermal delivery. Nat. Med. 8:415-419 (2002).

97. J. A. Matriano, M. Cormier, J. Johnson, W. A. Young, M. Buttery, K. Nyam, and P. E. Daddona. Macroflux microprojection array patch technology: a new and efficient approach for intracutaneous immunization. Pharm. Res. 19:63-70 (2002).

98. G. M. Glenn, M. Rao, G. R. Matyas, and C. R. Alving. Skin immunization made possible by cholera toxin. Nature 391:851 (1998).

99. G. M. Glenn, D. N. Taylor, X. Li, S. Frankel, A. Montemarano, and C. R. Alving. Transcutaneous immunization: a human vaccine delivery strategy using a patch. Nat. Med. 6:1403-1406 (2000).
100. F. Guerena-Burgueno, E. R. Hall, D. N. Taylor, F. J. Cassels, D. A. Scott, M. K. Wolf, Z. J. Roberts, G. V. Nesterova, C. R. Alving, and G. M. Glenn. Safety and immunogenicity of a prototype enterotoxigenic Escherichia coli vaccine administered transcutaneously. Infect. Immun. 70:1874-1880 (2002).

101. T. Scharton-Kersten, J. Yu, R. Vassell, D. O'Hagan, C. R. Alving, and G. M. Glenn. Transcutaneous immunization with bacterial ADP-ribosylating exotoxins, subunits, and unrelated adjuvants. Infect. Immun. 68:5306-5313 (2000).

102. R. Tierney, A. S. Beignon, R. Rappuoli, S. Muller, D. Sesardic, and C. D. Partidos. Transcutaneous immunization with tetanus toxoid and mutants of Escherichia coli heat-labile enterotoxin as adjuvants elicits strong protective antibody responses. $J$. Infect. Dis. 188:753-758 (2003).

103. M. Guebre-Xabier, S. A. Hammond, D. E. Epperson, J. Yu, L. Ellingsworth, and G. M. Glenn. Immunostimulant patch containing heat-labile enterotoxin from Escherichia coli enhances immune responses to injected influenza virus vaccine through activation of skin dendritic cells. J. Virol. 77:5218-5225 (2003).

104. G. Dziekan, D. Chisholm, B. Johns, J. Rovira, and Y. J. Hutin. The cost-effectiveness of policies for the safe and appropriate use of injection in healthcare settings. Bull. World Health Organ. 81:277-285 (2003). 